\title{
Evaluation of Rational use of Antibiotics in Medicine Ward of a Tertiary Care Hospital
}

\author{
Abubakar Siddique 1,3,*, Hafeez $\mathrm{AK}^{2,3}$, Shekhar $\mathrm{HS}^{3}$, Ashfaque $\mathrm{A}^{2,3}$ \\ 'Department of Pharmacy Practice, Unaizah College of Pharmacy, Qassim University, SAUDI ARABIA. \\ ²United Doctors Hospitals, Jeddah, SAUDI ARABIA. \\ ${ }^{3}$ Department of Pharmacy Practice, Visveswarapura Institute of Pharmaceutical Sciences, Bangalore-560070, Karnataka, INDIA.
}

\begin{abstract}
Background: Antibiotics are among the most frequently used drugs worldwide. An average $35 \%$ of the total health budget is spent on antibiotics. Overuse and/or misuse of antibiotics has significant consequences, such as increased cost, bacterial resistance, therapeutic failure, drug toxicity and drug interactions. The objective of this study was to evaluate the prescribing pattern of antibiotics in Medicine ward of a tertiary care hospital in Bangalore. Methods: Hospital based retrospective study carried out in medicine ward of a tertiary care hospital in Bangalore. A retrospective study was carried out by collecting the data of the medication orders given for a period of 12 months. A total of 741 medication order was studied of which 470 were for males and 271 for females, between the age group of 15-92 years. Results: The results showed that the most commonly prescribed class of antibiotics were: Cephalosporins 571 (47.22\%), Quinolone 237 (19.6\%), Cephalosporins + Penicillinase inhibitors 159 (13.15\%), Penicillin + Penicillinase inhibitor 73 (6.03), Aminoglycoside 27 (2.23\%), Macrolides 13 (1.07\%), Sulphonamides $1(0.08 \%)$, Tetracyclines $2(0.16 \%)$ and others 126 $(10.4 \%)$. Conclusion: The most commonly prescribed class of antibiotics was Cephalosporins. Detailed knowledge of antibiotic prescription pattern
\end{abstract}

is important to monitor the rational use of antibiotics and is important before the therapeutic policies and measures can be implemented. It's recommended to involve an active clinical pharmacist in the hospital to study the drug use pattern and intervention as and when necessary.

Key words: Prescribing, Antibiotics, Rational Use, Tertiary Care Hospital, Therapeutic Policies.

Correspondence

Abubakar Siddique

'Department of Pharmacy Practice, Unaizah College of Pharmacy, Qassim University, SAUDI ARABIA.

${ }^{3}$ Department of Pharmacy Practice, Visveswarapura Institute of Pharmaceutical Sciences, Bangalore-560070, Karnataka, India.

Phone: +966 534405236

Email: getabu2u@gmail.com

DOI: 10.5530/jyp.2020.12.70

\section{INTRODUCTION}

Antibiotics used effective in the treatment of infectious diseases due to their selective toxicity; that is, they have the main function to injure or kill an invading micro-organism without harming the cells of the host. In most instances the selective toxicity is relative rather than absolute, requiring that the concentration of the drug be carefully controlled to attack the micro-organism while still being tolerated by the host. ${ }^{1}$ where antibiotics are considered generally safe and effective in fighting diseases, but there are certain cases where antibiotics can actually be harmful and might cause numerous adverse effects. ${ }^{2}$

Antibiotics are most common used drugs worldwide. An average $35 \%$ of the overall health budget is spent on antibiotics in developed countries. Overuse and/or misuse of antibiotics has significant consequences, such as increased cost, bacterial resistance, therapeutic failure drug toxicity and drug interactions. ${ }^{3}$ In India, the cost of Antimicrobial agents is as high as $50 \%$ of the overall health budget and prevalence use of antimicrobials varies from $24-67 \%$, where as in the Duke University Medical Centre England it accounts for 34\%. Antimicrobials as a group contribute significantly to the cost of drugs and is claimed worldwide to account for $15-30 \%$ of total health budget. ${ }^{4,5}$

The Centres for Disease Control and Prevention (CDC) estimates that more than 100 million antibiotic prescriptions are written each year in the ambulatory care setting. Inappropriate antibiotic use will promote resistance. Antibiotic resistance has become a major problem worldwide. Resistance may result when antibiotics are used too often or inappropriately for viral infections. Antibiotic resistance has been described as a serious threat to global public health by the World Health Organization because there are now few and, in some cases, no antibiotics available to treat certain life threatening infections. ${ }^{6,7}$ Misuse of antimicrobial agents may increase the risks of drug resistant pathogens, side effects and costs of medical aid. The appropriate agent at the appropriate dose and dosing interval and right duration can achieve both a favourable clinical outcome and prevent the selection of resistance. Almost 20.0-50.0\% of antimicrobial use has been found to be questionable or inappropriate. Accurate information about prescribing patterns in hospitals is effective in improving the quality of antimicrobial prescriptions. $^{8-10}$

Selection of the most appropriate antibiotic requires knowledge of: the organism identity, susceptibility to a specific agent, site of infection, patient's factor and safety of the agent and value of the therapy. However, some critically ill patients require empiric therapy that is, immediate administration of the drug prior to bacterial identification and susceptibility testing. ${ }^{11}$ Appropriate drug utilization studies have are found to be crucial to evaluate whether drugs are properly used and utilized in terms of medical, social and economic aspects. Several professional societies have issued guidelines designed to reduce the use of antibiotics world- wide by means of various control strategies. Detailed knowledge of antibiotic prescription pattern is vital before the policies and measures can be implemented. Antibiotic guidelines and 
associated interventions are demonstrated to be effective in improving usage of antibiotics. ${ }^{12}$

Quality of life can be improved by enhancing standards of medical treatment at all levels of the health care delivery system. Setting standards and assessing the quality of care through performance review should become part of everyday clinical practice. The evaluation of prescribing patterns seeks to monitor, evaluate and suggest modifications in practitioners' prescribing habits so as to make rational, safe and effective pharmaceutical care and cost effective therapy. In this context, we determine our objective to evaluate the prescribing pattern of antibiotics in medicine ward of a Kempegowda Institute of Medical Sciences (KIMS) Hospital and Research centre in Bangalore.

\section{MATERIALS AND METHODS}

A hospital based retrospective descriptive study was carried out using medicine ward data records between January 2014- December 2014 on prescribing patterns of antibiotics from patients data collection case sheet and treatment charts from medical records department of KIMS Hospital and Research centre, Bangalore. A total 741 medication orders including at least one antibiotic given to the inpatients over a period of 12 months. These included the medications given to both males and females (470 and 271 respectively) in the age group of 15-92 years who were admitted to medicine wards of the hospital. Data was collected with respect to demographics of the patient, details of antibiotics prescribed (like class of antibiotic, route of administration, dose, duration etc) along with diagnosis made. The collected data was entered and analysed using Microsoft Excel 2007 version software to analyse prescribing pattern of antibiotics.

Table 1: Gender Distribution.

\begin{tabular}{ccc} 
Gender & Number & (\%)Percentage \\
\hline Male & 470 & 63.42 \\
Female & 271 & 36.57 \\
Total & 741 & 100 \\
Male: female ratio & $1.73: 1$ & \\
\hline
\end{tabular}

Table 2: Age Groups of the Patients (In Years).

\begin{tabular}{|c|c|c|}
\hline Age & Number & (\%)Percentage \\
\hline $15-20$ & 42 & 5.66 \\
\hline $21-30$ & 125 & 16.86 \\
\hline $31-40$ & 116 & 15.65 \\
\hline \multirow[t]{2}{*}{$41-50$} & \multicolumn{2}{|c|}{120} \\
\hline & \multicolumn{2}{|c|}{16.19} \\
\hline $51-60$ & 136 & 18.35 \\
\hline $61-70$ & 114 & 15.32 \\
\hline $71-80$ & 61 & 8.23 \\
\hline 81 and above & 27 & 3.64 \\
\hline Total & 741 & 100 \\
\hline Range & $15-92$ years & \\
\hline
\end{tabular}

Table 3: Hospitalization Stay By the Patients (Days).

\begin{tabular}{cc}
\hline Range & $1-41$ \\
Average stay & $7.48 \pm 2.8$ \\
\hline
\end{tabular}

\section{RESULTS}

741 patients admitted to the Internal Medicine ward were prescribed antibiotics during the study period. Their age group was between 15 to 92 years, their data were collected and analysed. Analysis of the data collected indicates that; the subjects involved were male $470(63.4 \%)$ and female $271(36.57 \%)$. This gives a male to female ratio of 1.73:1. (Table 1) The age distribution were: $15-20$ years $=42(5.66 \%) ; 21-30$ years $=$ $125(16.86 \%), 31-40$ years $=116(15.65 \%), 41-50$ years $=120(16.19 \%)$, $51-60$ years $=136(18.35 \%), 61-70$ years $=114(15.32 \%), 71-80$ years $=61(8.23 \%), 81-92$ years $=27(3.64 \%)$ Most of the subject involved in the study was between the age group of 51-60 years. The duration of hospitalization of the 741 patients was recorded. (Table 2). Average length of stay was $7.48 \pm 0.70$ days ranging from 1-41 days (Table 3 ).

In this study the diagnosis made were classified into Tuberculosis (TB) 115 (15.5\%), Lower respiratory tract infection (LRTI) 66 (8.9\%), Acute GE 45 (6.07\%), Urinary Tract Infection (UTI) 32 (4.3\%), HIV 19 (2.5\%), Upper Respiratory Tract Infection (URTI) 10 (1.3\%), Renal Infections 8 (1\%), Sexually Transmitted Disease (STD) 4 (0.5\%), Use of antibiotics as Prophylaxis 397 (53.5\%) and Miscellaneous infections 45 (6\%) (Figure 1) (Table 4).

Most common Route of Administration used was Intravenous route 875 (72.37\%) followed by Oral route $328(27.12 \%)$, Intramuscular route 5 $(0.43 \%)$ and Topical route $1(0.08 \%)$ (Table 5). Among 741 Patients, 225 (30.3\%) patients received Single antibiotic, $372(50.2 \%)$ patients received 2 antibiotics, 104 (14.4\%) patients received 3 antibiotics, 32 (4.32) patients received 4 antibiotics, $5(0.67 \%)$ patients received 5 antibiotics and 3 $(0.4 \%)$ patients received 6 antibiotics respectively during hospitalisation (Figure 2) (Table 6).

The commonly prescribed class of antibiotic were: Cephalosporins 571 (47.22\%), Quinolone 237 (19.6\%), Cephalosporins + Penicillinase inhibitors 159 (13.15\%), Penicillin + Penicillinase inhibitor 73 (6.03),

Table 4: Use of Antibiotics in Different Diagnosis in the Study.

\begin{tabular}{ccc}
\hline Diagnosis & Number & (\%)Percentage \\
\hline Tuberculosis (TB) & 115 & 15.5 \\
LRTI & 66 & 8.9 \\
Acute GE & 45 & 6.0 \\
UTI & 32 & 4.3 \\
HIV & 19 & 2.5 \\
URTI & 10 & 1.3 \\
Renal Infection & 8 & 1.0 \\
STD & 4 & 0.5 \\
Prophylaxis & 397 & 53.5 \\
Miscellaneous & 45 & 6.0 \\
Total & 741 & 100 \\
\hline
\end{tabular}

Table 5: Route of Administration of Drugs.

\begin{tabular}{ccc}
\hline Route & Number & (\%) Percentage \\
\hline Intravenous & 875 & 72.3 \\
Oral & 328 & 27.1 \\
Intramuscular & 5 & 0.43 \\
Topical & 1 & 0.08 \\
Total & 1209 & 100 \\
\hline
\end{tabular}


Aminoglycoside 27 (2.23\%), Macrolides 13 (1.07\%), Tetracyclines $2(0.16 \%)$, Sulphonamides $1(0.08 \%)$ and others $126(10.4 \%)$. The most commonly prescribed antibiotic was Cephalosporins (Figure 3) (Table 7).

Among Cephalosporins: Ceftriaxone 460 (63.44\%), Ceftriaxone + Sulbactam 146 (20.13), Cefixime 92 (12.68\%), Ceftriaxone + Tazobactam $13(1.79 \%)$ and Cefpodoxime $6(0.82 \%)$, Cefotaxim 4 (0.55\%), Cefurexime 3 (0.41\%), Cefadroxil 1 (0.13\%) were used. Hence Ceftriaxone was the most commonly prescribed antibiotic in the present study (Figure 4).

Among Quinolones (237); Ciprofloxacin 134 (56.54\%), Levofloxacin 50 (21.09\%), Moxifloxacin 3 (16.45\%), Ofloxacin 39 (16.45\%), Ciprofloxacin + Tinidazole (Anti-diarrheals) 6 (2.5\%) and Sparfloxacin 5 (2.1\%) (Figure 5). Among Penicillins (73): Pipericilline + Tazobactam $51(69.86 \%)$ and Amoxicillin + Clavalunic acid 22 (30.13\%) (Figure 6). Among Aminoglycosides (27), Amikacin 20 (76.9\%) and Gentamicin 6 (23.07\%) were used (Figure 7). In Macrolides (13); Azithromycin 7 (53.8\%) and Clarithromycin 6 (46.15\%) were used (Figure 8).

In Sulphonamides Co-trimoxazole was prescribed in 3 prescriptions. Among Tetracyclines, Doxycycline was prescribed in 3 prescriptions. Other antibiotics (126) used were included Metronidazole 121 (93.7\%), Streptomycin 5 (3.8\%), Linidazoline 1 (0.77\%), Nitrofurantoin $1(0.77 \%)$ and Rifampicin 1 (0.77\%) (Figure 9).

\section{DISCUSSION}

Antibiotics represent one of the most commonly used drugs. Their irrational use leads to a number of consequences in terms of cost, side

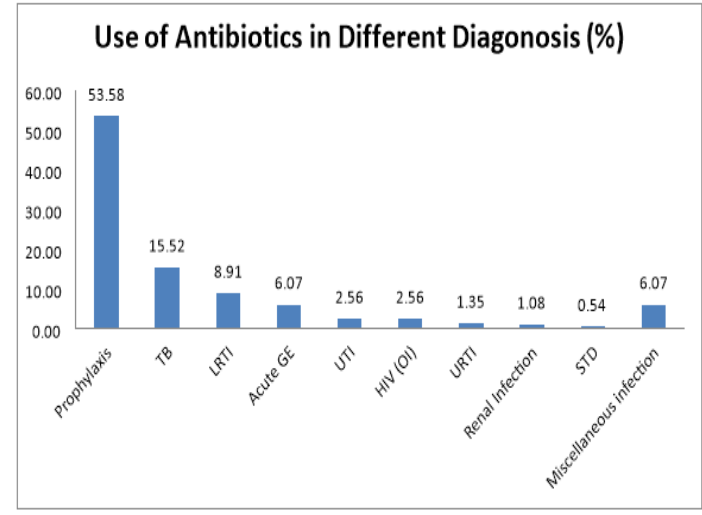

Figure 1: Diagnosis and treatment details.

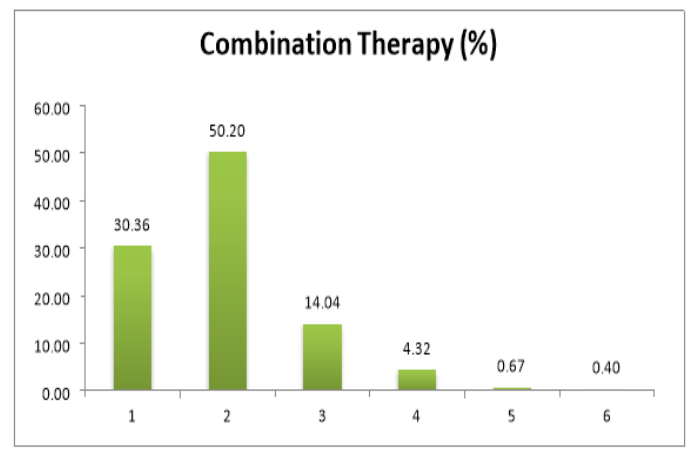

1-Single antibiotic therapy, 2-Two antibiotic therapy, 3-Three antibiotic therapy, 4-Four antibiotic therapy, 5- Five antibiotic therapy, 6-Six antibiotic therapy.

Figure 2: Usage of antibiotics combination therapy. effects and development of bacterial resistance. Antibiotic resistance among pathogenic micro-organisms is a matter of worldwide concern. Selective pressure by antimicrobial drugs is by far the most important driving force for the development of such resistance. Around 30\% of the hospitalized patients are treated with Antibiotics. Third-generation cephalosporins were used mostly for inpatients with hospital-acquired infections because of their broad spectrum and activity. Consequently, the true frequency and proportion of antibiotic use may have been underestimated.

Although causal inferences cannot be made in the present study, there are some underlying public beliefs that may explain the high level of antibiotic use such as doctors' (or patients') poor awareness of the dire consequences of overuse of antibiotics, irrational use of antibiotics due to financial incentives and profit driven prescribing behaviours and no effective supervisory measures for antibiotic utilization. Further studies should be conducted to investigate reasons for overuse of antibiotics and how to reduce the high-use rate. ${ }^{13}$ The essential drugs may be limited in number but they should be carefully selected based on the clinical guidelines. The development of treatment guidelines and essential drugs list are of more importance in resource poor situations where the availability of drugs in the public sector is often erratic. The World Health Organization (WHO) is advocating the promotion of rational use of drugs by promoting the implementation of standard treatment guidelines and essential drugs. The process for guideline development should be aimed at identifying intervention that will ensure the best possible health outcomes. The purpose of treatment guideline is to encourage the treatment that offers individual patients maximum

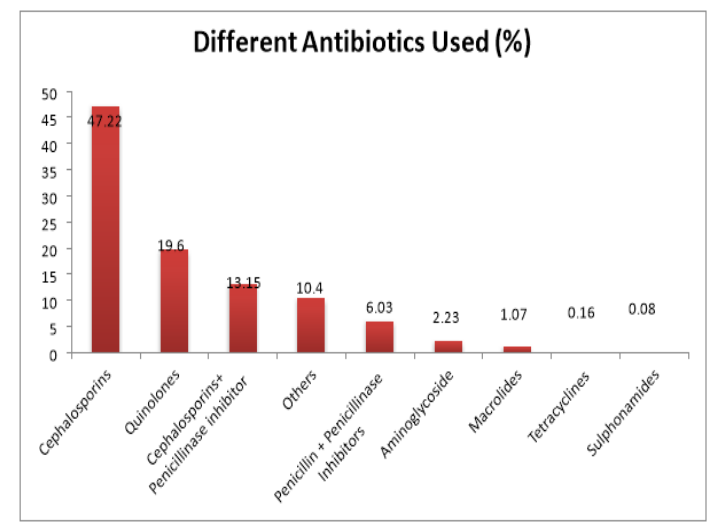

Figure 3: Different antibiotics agents used in our study.

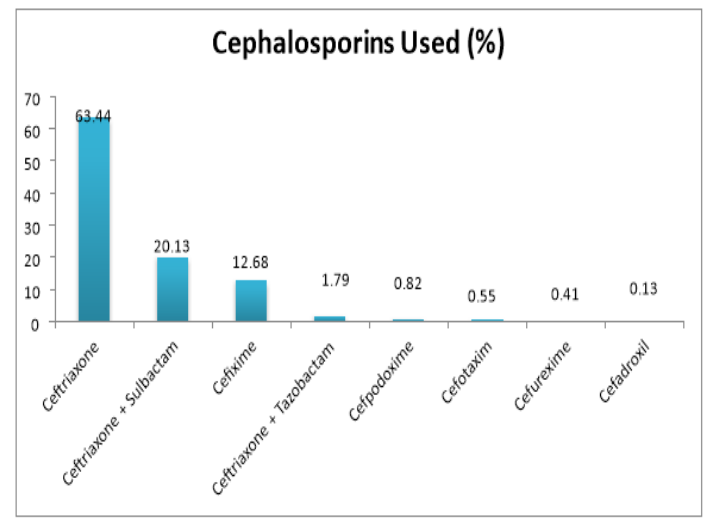

Figure 4: Cephalosporins class of Antibiotics used in our study. 
Table 6: Details of Antibiotics Therapy.

\begin{tabular}{ccc}
\hline Therapy & Number & (\%)Percentage \\
\hline Single Antibiotic & 225 & 30.3 \\
Two Antibiotic & 372 & 50.2 \\
Three Antibiotic & 104 & 14.0 \\
Four Antibiotic & 32 & 4.32 \\
Five Antibiotic & 5 & 0.67 \\
Six Antibiotic & 3 & 0.4 \\
Total & 741 & 100 \\
\hline
\end{tabular}

Table 7: Types of Antibiotics Used In the Study.

\begin{tabular}{ccc}
\hline Antibiotics Type/Class & Number & (\%)Percentage \\
\hline Cephalosporins & 571 & 47.22 \\
Quinolones & 237 & 19.6 \\
Cephalosporins + Penicillinase & 159 & 13.1 \\
inhibitors & & \\
Pencillin + penicillinase inhibitors & 73 & 10.4 \\
Aminoglycoside & 27 & 6.03 \\
Macrolides & 13 & 2.23 \\
Tetracyclines & 2 & 1.07 \\
Sulphonamides & 1 & 0.16 \\
Others & 126 & 0.08 \\
Total & 1209 & 100 \\
\hline
\end{tabular}

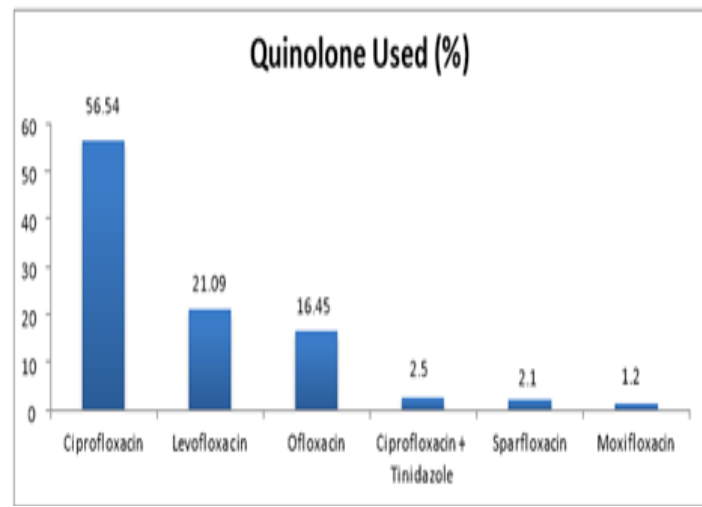

Figure 5: Quinolone group of antibiotics used in our study.

likelihood of benefit and minimum harm and is acceptable in terms of cost. $^{14}$

The standard treatment guidelines and essential drugs are the basic tools for assisting health professionals to choose the most appropriate medicine for the given patient with a given condition. It should be followed by the appropriate use of the selected medicine. Health care providers and those responsible for dispensing medicines should take every opportunity to inform patients about the rational use of drugs, including the use of drugs for self-medication at the time they are dispensed. ${ }^{15}$

Sumit Patel, et al. conducted a study on prevailing scenario of fixeddose drug combinations (FDCs) for essentiality and rationality of drug combinations in Indian drug review (IDR). Upon evaluation they found the highest number of drugs and FDCs were found in category of antimicrobial drugs and they considered there results analysis about

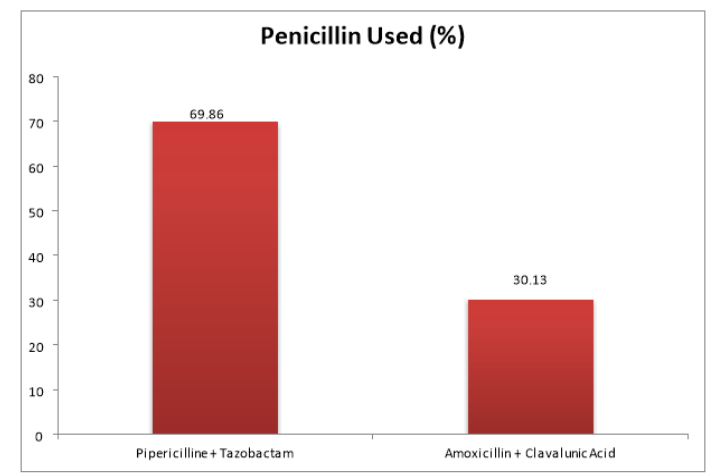

Figure 6: Penicillin class of Antibiotics used in our study.

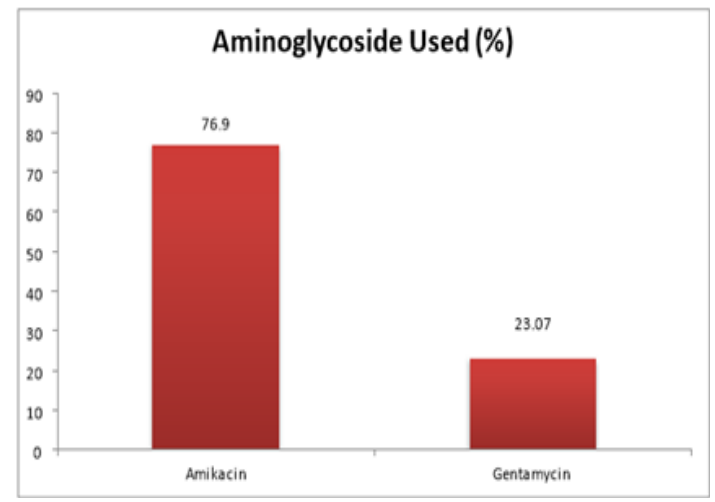

Figure 7: Aminoglycosides class of Antibiotics used in our study.

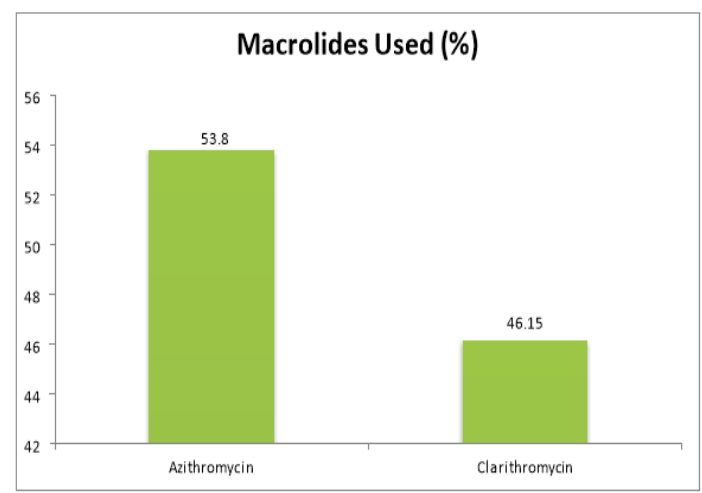

Figure 8: Macrolides class of antibiotics used in our study.

$8.80 \%$ FDCs were found to be rational, around $19.37 \%$ categorized as semi rational and remaining $71.83 \%$ FDCs were considered irrational in their study. ${ }^{16}$ Considering the appropriate use of antibiotic and the prescribers should remember that rational use of antibiotics always have better outcomes for the patients as well as practicing doctors. As much as possible fair choice of antibiotic is important. Antibiotics which used for the prophylaxis, in general and particularly in surgery, requires significant thinking and it should be evidence based in practice. ${ }^{17}$

Several international reports on the subject of antimicrobial resistance since 1998 had acknowledged the important role clinical pharmacists have to play in influencing promising foundation to promote rational use of antibiotics. ${ }^{18}$ Clinical pharmacy service is to optimize pharmacotherapy outcomes by working to achieve the best possible quality use of medicines. ${ }^{19}$ Indefinitely the clinical pharmacy activities 


\section{Other Antibiotics (\%)}

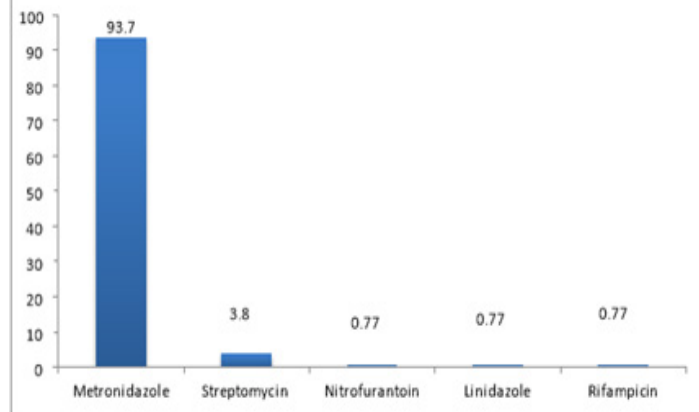

Figure 9: Miscellaneous classes of antibiotics used in our studies.

reduce hospitalization due to drug-related problems, probability of readmission to the hospital and total cost of drug therapy. ${ }^{20}$

Quickening the availability of culture and sensitivity reports will enable the treatment to have a sound bacteriological basis. Antibiotic resistance is becoming a problem and formulation of a hospital antibiotic use policy is a matter of urgent concern. An educational programme and an antibiotic order form may be useful initiatives to reduce antibiotic use. Guidelines for antibiotic use in the community and restricting the level of health care practitioners who can prescribe antibiotics are required. ${ }^{21}$ hence detailed evaluation and knowledge of antibiotic prescription pattern is important before the policies and measures can be implemented.

\section{CONCLUSION}

Broad-spectrum Cephalosporins (ceftriaxone) were more commonly used than narrow spectrum ones in our study. An obvious advantage of broad-spectrum antibiotics means least requirement for identification of the infecting pathogen in comparison with narrow spectrum antibiotics before treatment. More than $90 \%$ of the antibiotics were prescribed by brand names and more than $70 \%$ of the antibiotics were administered by parenteral route and it was also found that more than $80 \%$ of the patients studied belonged to lower and lower middle socio-economic status. So cost is the matter of major concern in this study. Pharmacoeconomics plays an important role in rational therapeutic decision making.In this study, it was concluded that more than $50 \%$ of antibiotics prescribed as prophylaxis. Availability of culture and sensitivity reports will enable the treatment to have a sound bacteriological basis. Antibiotic resistance is becoming a problem and formulation of a hospital antibiotic use policy is a matter of urgent concern. Hence detailed knowledge of antibiotic prescription pattern is important before the policies and measures can be implemented. An agreed clinical guideline helps in the selection of essential drugs. The interventional programmes and policies should be formulated to promote infectious control with rational use of antibiotics aimed to minimize the emergence of bacterial resistance, reduce total antibiotic use and futile expenses. A clinical Pharmacist would play a key role in antibiotic audit and rational use of antibiotics in the hospital as he has a thorough knowledge regarding the drugs. It's recommended to involve an active clinical pharmacist in the various wards of hospitals to study the drug use pattern and intervention as and when necessary.

\section{ACKNOWLEDGEMENT}

The authors disclose no potential bias or conflict of interest relating to this study.We are thankful to Medical record department of KIMS
Hospital and Research centre for providing access to data from patient record files.

\section{CONFLICT OF INTEREST}

The authors declare that there is no conflict of interest.

\section{ABBREVIATIONS}

CDC: Centres for Disease Control and Prevention, KIMS: Kempegowda Institute of Medical Sciences, WHO: World Health Organization, FDCs: fixed-dose drug combinations, IDR: Indian drug review

\section{REFERENCES}

1. Harvey RA, Champe PC. Lippincott's Illustrated reviews. $3^{\text {rd }}$ edi. William and Wilkins. 2005;341-52.

2. Rhonda $H$, Arlene P, Kathy B, Lianne D. The Association for Professionals in Infection Control and Epidemiology (APIC).2005. www.apic.org. [Cited 2020 Feb 15]: Brochure. Available from: https://www.mysciencework.com/ publication/download/use-of-resequencing-oligonucleotide-microarrays-foridentification-of-streptococcus-pyogenes-and-associated-antibiotic-resistanc/ $\mathrm{f} 4 \mathrm{c5c9b9d7c59666f5fd8fa00f926407}$

3. Zulal O, Serpil E, Ayten K, Mustafa E, Kemalettin O, Mehmet AT. Changes in antibiotic use, cost and consumption after an antibiotic restriction policy applied by infectious disease specialists. Jpn J Infect Dis. 2005;58(6):338-43.

4. Kunin CM. Rational use of antibiotics. WHO Drug Information. 1990;4(1):4-7.

5. Adiga MNS, Alwar MC, Pai MRSM, Adiga US. Pattern of antimicrobial agents use in hospital deliveries: A prospective comparative study. Online $\mathrm{J}$ Health Allied Scs. 2009;8(4):10

6. McCaig LF, Besser RE, Hughes JM. Antimicrobial drug prescription in ambulatory care settings, United States, 1992-2000 [Published correction appears in Emerg Infect Dis 2003; 9:609]. Emerg Infect Dis. 2003;9(4):432-7.

7. World Health Organisation: Antimicrobial resistance: The facts. In WHO Essential Drugs Monitor No. 28 and 29 edition. Geneava. 2000;8-9.

8. Guven GS, Uzun O. Principles of good use of antibiotics in hospitals. J Hosp Infect. 2003;53(2):91-6.

9. Hecker MT, Aron DC, Patel NP, Lehmann MK, Donskey CJ. Unnecessary use of antimicrobials in hospitalized patients. Arch Intern Med. 2003;163(8):972-8.

10. Paudel KR, Sharma M, Das BP. Prevalence of antimicrobial chemotherapy in hospitalized patients in the department of internal medicine in a tertiary care center. Nepal Med Coll J. 2008;10(2):91-5.

11. Whalen K, Richard F, Thomas A. Panavelil. Lippincott Illustrated Reviews: Pharmacology. $6^{\text {th }}$ ed. Philadelphia, PA: Wolters Kluwer. 2015

12. Harvey K, Dartnell J, Hemming M: Improving antibiotic use: 25 years of antibiotic guidelines and related initiatives. Commun Dis Intell. 2003;27:9-11.

13. Vander MJW, Gyssens IC: Quality of antimicrobial drug prescription in hospital Clin Microbiol Infect. 2001;7(Suppl):12-5.

14. WHO. Drugs and Therapeutics Committees - A Practical Guide, World Health Organization. 2003

15. Khan NA, Abid M, Maheshwari KK, Kaviarasan PK, Mohanta GP. Antibiotic prescribing pattern in department of dermatology of a teaching hospital in Tamil Nadu. Indian J Pharm Pract. 2010;3(3)

16. Sumit P, Rima S, Sagun D. A study of prevailing scenario of fixed dose combinations available in Indian market. Int J Pharm. 2015;5:1155-63.

17. Desai SV. Rational use of antibiotics in clinical practice: Pharmacological considerations. J Integr Health Sci. 2019;7(1):1-2.

18. Kieran $\mathrm{H}$. Antibiotic pharmacists in the ascendancy. J Antimicrob Chemother. 2007;60(1):i73-6.

19. Dooley M, Bogovic A, Carroll M, Cuell S, Galbraith K, Matthews H. SHPA Standards of Practice for Clinical Pharmacy. J Pharm Pract Res. 2005;35(2):12246.

20. Dooley MJ, et al. A Prospective Multicenter Study of Pharmacist Initiated Changes to Drug Therapy and Patient Management in Acute Care Government Funded Hospitals. Br J Clin Pharmacol. 2003;57(4):513-21.

21. Shankar RP, Partha P, Shenoy NK, Easow JM, Brahmadathan KN. Prescribing patterns of antibiotics and sensitivity patterns of common microorganisms in the Internal Medicine ward of a teaching hospital in Western Nepal: A prospective study. Ann Clin Microbiol Antimicrob. 2003;2(1):7. 